\title{
GENERALIZED VELOCITY-DENSITY MODEL BASED ON MICROSCOPIC TRAFFIC SIMULATION
}

\author{
Oussama Derbel ${ }^{1}$, Tamás Péter ${ }^{2}$, Benjamin Mourllion ${ }^{3}$, Michel Basset ${ }^{4}$ \\ ${ }^{1,3,4}$ MIPS Laboratory (Modelling, Intelligence, Process and Systems), \\ Haute Alsace University, Mulhouse, France \\ ${ }^{2}$ Dept of Control for Transportation and Vehicle Systems, \\ Budapest University of Technology and Economics, Hungary
}

Submitted 1 September 2014; resubmitted 10 February 2015, 7 July 2015, 21 February 2016; accepted 15 March 2016; published online 12 April 2017

\begin{abstract}
In case of the Intelligent Driver Model (IDM) the actual Velocity-Density law $V(D)$ applied by this dynamic system is not defined, only the dynamic behaviour of the vehicles/drivers is determined. Therefore, the logical question is whether the related investigations enhance an existing and known law or reveal a new connection. Specifically, which function class/type is enhanced by the IDM? The publication presents a model analysis, the goal of which was the exploration of a feature of the IDM, which, as yet, 'remained hidden'. The theoretical model results are useful, this analysis important in the practice in the field of hybrid control as well. The transfer of the IDM groups through large-scale networks has special practical significance. For example, in convoys, groups of special vehicle, safety measures with delegations. In this case, the large-scale network traffic characteristics and the IDM traffic characteristics should be taken into account simultaneously. Important characteristics are the speed-density laws. In case of effective modelling of large networks macroscopic models are used, however the IDMs are microscopic. With careful modelling, we cannot be in contradiction with the application of speed-density law, where there IDM convoy passes. Therefore, in terms of practical applications, it is important to recognize what kind of speed-density law is applied by the IDM convoys in traffic. Therefore, in our case the goal was not the validation of the model, but the exploration of a further feature of the validated model. The separate validation of the model was not necessary, since many validated applications for this model have been demonstrated in practice. In our calculations, also the applied model parameter values remained in the range of the model parameters used in the literature. This paper presents a new approach for Velocity-Density Model (VDM) synthesis. It consists in modelling separately each of the density and the velocity (macroscopic parameter). From this study, safety time headway (microscopic parameter) can be identified from macroscopic data by mean of interpolation method in the developed map of velocity-density. By combining the density and the velocity models, a generalized new VDM is developed. It is shown that from this one, some literature VDMs, as well as their properties, can be derived by fixing some of its parameters.
\end{abstract}

Keywords: velocity-density model; microscopic traffic simulation; adaptive cruise control.

\section{Introduction}

Traffic modelling can be classified into the three following classes.

The first class includes the microscopic models where the traffic is viewed as a system of interacting particles or vehicles. This interaction is modelled by differential equations as done by Bando et al. (1998), Helbing and Tilch (1998), Treiber et al. (2000b), Molina (2005), Ge et al. (2008), Kesting (2008), Rakha and Gao (2010). For interested readers, a general overview of microscopic traffic models is presented by Derbel et al. (2012a, 2012b).
The second class includes the macroscopic models where the traffic is viewed as one group of particles. Macroscopic parameters are traffic density, traffic flow and velocity, which are used for graphic representation of the fundamental diagram. Several works are based on this approach such as those ones developed by Holden and Risebro (1995), Herty and Klar (2003), Treiber et al. (2000b), Péter (2012), Bede et al. (2013), Dömötörfi et al. (2016), Péter et al. (2015), Bede and Péter (2014).

The third class includes the mesoscopic models, which appears to be an intermediate between the two last classes. Here, the traffic is viewed as clusters of ve-

Corresponding author: Oussama Derbel

E-mail: oussama.derbel@gmail.com 
hicles. Several studies have been realized in this field such as those done by Prigogine and Herman (1971), Paveri-Fontana (1975), Mahnke and Kaupužs (1999).

The presented research work deals with microscopic and macroscopic approaches, where the traffic is represented by one cluster. The goal is to predict traffic density and velocity with a minimum of errors. Most of the literature Velocity-Density Models (VDMs) are developed and identified by mean of experimental macroscopic traffic data. Since we do not have these data, our work is based on the simulation data. Here, the idea is to generate macroscopic traffic data from microscopic traffic model simulation. Nonetheless, this microscopic model must faithfully reproduce the traffic behaviours and be validated by the fundamental diagram study.

In this paper, the developed VDM is based on the Intelligent Driver Model (IDM) (Kesting 2008; Treiber et al. 2000a). Our new method proceeds by the identification of the simulated velocity and density data separately. Among the advantages of this method is the ability to identify each microscopic parameter from macroscopic ones by interpolation method using our developed map of macroscopic data related to microscopic parameter. Then, a new VDM is computed with a mathematical development of the velocity and the density models. In addition, it will be shown later that some of existing models in the literature, as well as their properties can be derived from this new VDM by fixing some of its parameters.

In this paper, Section 1 presents a brief state-of-the art of the existing VDMs. Section 2 identifies the velocity and the density functions. Section 3 presents the synthesis method of the new VDM. Section 4 presents the generalized VDM and the last section concludes and gives outlooks.

\section{State-of-the-art of the Velocity-Density Models}

Two classes of VDMs can be distinguished: the stochastic models and the deterministic models. This section presents a brief state-of-the-art of deterministic VDMs considering the chronological order.

\subsection{Greenshields Model}

Up to our knowledge, the first deterministic VDM was proposed by Greenshields (1935). This affine model is given by the following expression:

$$
V(D)=V_{\max }\left(1-\frac{D}{D_{\max }}\right),
$$

where: $D_{\max }$ is the maximum density which is the jam density.

This model is identified by linear regression method using seven experimental observations.

Recently, Wang et al. (2013) have showed that, with more than seven data, the Greenshields model is not enabling the prediction of velocity and density.

\subsection{Greenberg Model}

By the analogy with fluid flow, Greenberg (1959) has developed a logarithmic velocity-density relationship given by:

$$
V(D)=V_{\max } \log \left(\frac{D}{D_{\max }}\right)
$$

The main drawback of this model is its inability to predict velocities for low densities. Indeed, the velocity tends to infinity when the density tends to zero, which is unrealistic.

\subsection{Underwood Model}

Underwood (1961) has derived an exponential model in order to overcome the drawbacks of the Greenberg and Greenshields models for the free traffic flow condition. This model is given by:

$$
V(D)=V_{\max } \exp \left(-\frac{D}{D_{\max }}\right) .
$$

The main drawback of the Underwood model is that velocity becomes zero only when density $D$ reaches infinity and not $D=D_{\max }$. Hence, this model cannot be used for predicting velocities at high densities.

\subsection{Newell Model}

The VDM of Newell (1961) is expressed by:

$$
V(D)=V_{\max }\left(1-\exp \left(-\frac{\lambda}{V_{\max }}\left(\frac{1}{D}-\frac{1}{D_{\max }}\right)\right),\right.
$$

where: $\lambda>0$ is the slope of inter-distance-velocity curve at $V=0 \mathrm{~km} / \mathrm{h}$.

Here, when $D=D_{\max }$, then $V=0 \mathrm{~km} / \mathrm{h} ; D=0$ then $V=V_{\max }$. Therefore, the limit conditions are verified by the Newell model.

\subsection{Drake Model}

Drake et al. (1967) have enhanced the Greenberg model by studying various macroscopic traffic models. By estimating the density from velocity and flow data, they propose the new VDM expressed by:

$$
V(D)=V_{\max } \exp \left(-\frac{1}{2}\left(\frac{D}{D_{\max }}\right)^{2}\right)
$$

According to Ardekani et al. (2011), this model presents a better fitting than the models of Greenshields, Greenberg and Underwood for non-congested conditions. In case of congested conditions, the Drake model presents a poor data fitting.

\subsection{Pipes Model}

Pipes (1967) has generalized the Greenshields model leading to a new velocity-density relationship given by:

$$
V(D)=V_{\max }\left(1-\left(\frac{D}{D_{\max }}\right)^{r}\right)^{m}
$$


By varying the values of $r$ and $m$, a family of models can be developed. For example, Greenshields model is obtained for $r=1$ and $m=1$.

\subsection{Drew Model}

Drew (1968) has proposed another model expressed as follows:

$$
V(D)=V_{\max }\left(1-\left(\frac{D}{D_{\max }}\right)^{\frac{p+1}{2}}\right)
$$

According to Ardekani et al. (2011), at free-flow phase, the Drew model presents an underestimated velocity, but in the congested phase, the velocity is overestimated.

\subsection{Del Castillo Model}

Del Castillo and Benítez (1995) have developed a VDM, which is given by the following expression:

$$
V(D)=V_{\max }\left(1-\exp \left(\frac{\left|C_{j}\right|}{V_{\max }}\left(1-\frac{D_{\max }}{D}\right)\right),\right.
$$

where: $C_{j}$ is the kinematic wave speed given by:

$$
C_{j}=\left.D_{\max } \frac{d V}{d D}\right|_{D=D_{\max }} .
$$

According to MacNicholas (2008), the drawback of this model is the large kinematic wave speed range, which makes difficult its estimation. model.

If $D_{\max }=1$ and $C_{j}=\lambda$, then we have the Newell

\subsection{Van Aerde Model}

Van Aerde (1995) developed a new VDM, which is based on a simple car-following model. This last one depends on the free and current velocity and a calibrated constant. The VDM is given by:

$$
D(V)=\frac{1}{C_{1}+\frac{C_{2}}{V_{\max }-V}+C_{3} V},
$$

where: $C_{1}, C_{2}, C_{3}$ are constants which can be calibrated by nonlinear regression.

\subsection{MacNicholas Model}

MacNicholas (2008) have proposed the following VDM:

$$
V(D)=V_{\max } \frac{D_{\max }^{q}-D^{q}}{D_{\max }^{q}+m D^{q}},
$$

where: $m, q$ are real constants.

By varying these constants, a family of models can be developed. For example, if $D_{\max }=1, m=0$ and $q=\frac{p+1}{2}$ the Drew model expression is found.

\subsection{Power Function Model}

The power function proposed by Del Castillo (2012) is given by:

$V(D)=\frac{1}{\rho}\left(b+(a-b) \rho-\left((a \rho)^{\Theta}+(b(1-\rho))^{\Theta}\right)^{\frac{1}{\Theta}}\right)$,

where: $\theta$ is a shape parameter; $a, b$ are constants;

$\rho=\frac{D}{D_{\max }}$.

\subsection{Exponential Model}

The exponential model is given by Del Castillo (2012) as follows:

$V(D)=\frac{1}{\rho}\left(b+(a-b) \rho-\frac{1}{\alpha} \log \left(e^{\alpha \rho a}+e^{\alpha b(1-\rho)}\right)-1\right)$,

where:

$$
a=\frac{V_{\max }}{-C_{j}\left(1-e^{\alpha b}\right)} ; \quad b=\frac{1}{\left(1-e^{\alpha b}\right)} .
$$

\subsection{Negative Power Model}

The negative power model is presented by Del Castillo (2012) as:

$$
V(D)=\frac{1}{\rho} \cdot\left(\left(u_{f} \cdot \rho\right)^{-\Theta}+(1-\rho)^{-\Theta}\right)^{\frac{1}{\Theta}} .
$$

\subsection{Environmental Parameterization Model}

Péter and Fazekas (2014) have enhanced the environmental parameterization model by studying various macroscopic traffic models. The classical literature does not deal with the definition of the environmental vector, but the velocity is determined not only by vehicle density, but by other environmental parameterization, as well: this refinement can be implemented with the modification of $V_{\max }$, or via the modification of the function itself considering the weather, visibility, road quality, width of the road. These environmental, seasonal factors can be represented in the environmental parameter vector $\underline{e}: V(\rho, \underline{e})$ :

$$
V(\rho, \underline{e})=\frac{e_{4} V_{\max }}{e_{3}+e_{2}\left(\frac{\rho}{1-\rho^{e_{5}}}\right)^{e_{1}}} .
$$

In this case, the parameter vector $\underline{e}$ contains 5 parameters (Fig. 1).

The Table 1 demonstrates the favourable and unfavourable parameter domains. The internal domain is located between the two distinct domains, in most of the cases the practical parameter comes from this internal interval. The borders of the intervals are empirical values, in a given case the coordinates of the $\underline{e}=\left[e_{1}, e_{2}, e_{3}\right.$, $\left.e_{4}, e_{5}\right]$ parameter-vector are determined via regression analysis after the velocity-density measurement.

The specialty of the introduced $V(\rho, \underline{e})$ function is that it gives the same results as the linear function of Greenshields, if every parameters' value equals to 1 (Fig. 2). 


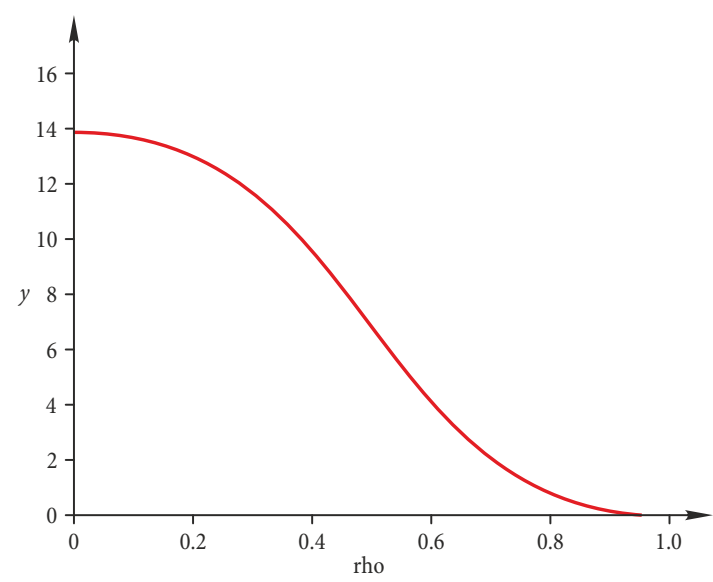

Fig. 1. $V(\rho)$ velocity-density function with $e_{1}=2 ; e_{2}=1$; $e_{3}=1 ; e_{4}=1 ; e_{5}=1$ parameters

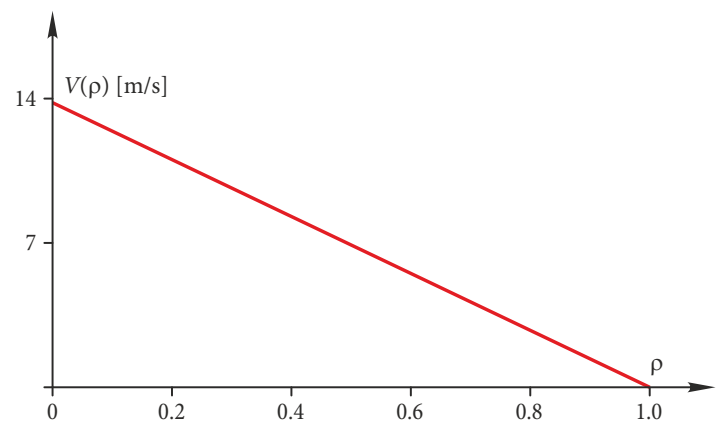

Fig. 2. $V(\rho)$ velocity-density function with $e_{1}=1 ; e_{2}=1$; $e_{3}=1 ; e_{4}=1 ; e_{5}=1$ parameters

Table 1. Demonstration of the $e$ parameter vector

\begin{tabular}{|l|l|l|l|}
\hline$e_{i}$ & $\begin{array}{c}\text { Meaning of the } \\
\text { parameter }\end{array}$ & \multicolumn{1}{|c|}{$\begin{array}{c}\text { Unfavourable } \\
\text { cases }\end{array}$} & Favourable cases \\
\hline$e_{1}$ & Road quality & Bad: $e_{1}=0.1-0.3$ & Good: $e_{1}=3-4$ \\
\hline$e_{2}$ & Curly road & $\begin{array}{l}\text { Lot of curves: } \\
e_{2}=3-4\end{array}$ & $\begin{array}{l}\text { Few curves: } \\
e_{2}=0.1-0.2\end{array}$ \\
\hline$e_{3}$ & Slippery road & $\begin{array}{l}\text { Bad, slippery: } \\
e_{3}=1.2-4\end{array}$ & No slippery: $e_{3}<1$ \\
\hline$e_{4}$ & Safety, visibility & $\begin{array}{l}\text { Bad conditions: } \\
e_{4}=0.5-0.7\end{array}$ & $\begin{array}{l}\text { Good conditions: } \\
e_{4}>1\end{array}$ \\
\hline$e_{5}$ & Width of road & $\begin{array}{l}\text { Narrow: } \\
e_{5}=0.1-0.2\end{array}$ & Wide: $e_{5}>4$ \\
\hline
\end{tabular}

This result shows that Greenshields' linear function has parameter values from the mid-range, so that provides really an average $V(\rho)$ velocity-density function relationship in practice.

\subsection{Synthesis}

Table 2 summarizes the references with the macroscopic traffic models cited in this section. $V_{\max }$ is the maximum velocity, $D_{\max }$ the jam density, $\lambda$ the slope of inter-distance-velocity curve at $V=0, C_{1}, C_{2}, C_{3}, m, p, q$ and $r$ are constants and $C_{j}$ is the kinematic wave speed at jam density.
Table 2. VDM summary

\begin{tabular}{|c|c|}
\hline Reference & Model \\
\hline $\begin{array}{l}\text { Greenshields } \\
(1935)\end{array}$ & $V(D)=V_{\max }\left(1-\frac{D}{D_{\max }}\right)$ \\
\hline $\begin{array}{l}\text { Greenberg } \\
(1959)\end{array}$ & $V(D)=V_{\max } \log \left(\frac{D}{D_{\max }}\right)$ \\
\hline $\begin{array}{l}\text { Underwood } \\
(1961)\end{array}$ & $V(D)=V_{\max } \exp \left(-\frac{D}{D_{\max }}\right)$ \\
\hline $\begin{array}{l}\text { Newell } \\
(1961)\end{array}$ & $V(D)=V_{\max }\left(1-\exp \left(-\frac{\lambda}{V_{\max }}\left(\frac{1}{D}-\frac{1}{D_{\max }}\right)\right)\right)$ \\
\hline $\begin{array}{l}\text { Drake et al. } \\
(1967)\end{array}$ & $V(D)=V_{\max } \exp \left(-\frac{1}{2}\left(\frac{D}{D_{\max }}\right)^{2}\right)$ \\
\hline Pipes (1967) & $V(D)=V_{\max }\left(1-\left(\frac{D}{D_{\max }}\right)^{\prime}\right)$ \\
\hline Drew (168) & $V(D)=V_{\max }\left(1-\left(\frac{D}{D_{\max }}\right)^{\frac{p+1}{2}}\right)$ \\
\hline $\begin{array}{l}\text { Del Castillo } \\
\text { and Benítez } \\
\text { (1995) }\end{array}$ & $V(D)=V_{\text {Max }}\left(1-\exp \left(\frac{\left|C_{j}\right|}{V_{\max }}\left(1-\frac{D_{\max }}{D}\right)\right)\right.$ \\
\hline $\begin{array}{l}\text { Van Aerde } \\
\text { (1995) }\end{array}$ & $D(V)=\frac{1}{C_{1}+\frac{C_{2}}{V_{\max }-V}+C_{3} V}$ \\
\hline $\begin{array}{l}\text { MacNicholas } \\
(2008)\end{array}$ & $V(D)=V_{\text {Max }} \frac{D_{\max }^{q}-D^{q}}{D_{\max }^{q}+m D^{q}}$ \\
\hline $\begin{array}{l}\text { Del Castillo } \\
\text { (2012) } \\
\text { (power } \\
\text { model) }\end{array}$ & $V(D)=\frac{1}{\rho}\left(b+(a-b) \rho-\left((a \rho)^{\Theta}+(b(1-\rho))^{\Theta}\right)^{\frac{1}{\Theta}}\right.$ \\
\hline $\begin{array}{l}\text { Del Castillo } \\
\text { (2012) } \\
\text { (exponential } \\
\text { model) } \\
\end{array}$ & $V(D)=\frac{1}{\rho}\left(b+(a-b) \rho-\frac{1}{\alpha} \log \left(e^{\alpha \rho a}+e^{\alpha b(1-\rho)}\right)-1\right.$ \\
\hline $\begin{array}{l}\text { Del Castillo } \\
\text { (2012) } \\
\text { (negative } \\
\text { power } \\
\text { model) } \\
\end{array}$ & $V(D)=\frac{1}{\rho}\left(\left(u_{f} \rho\right)^{-\Theta}+(1-\rho)^{-\Theta}\right)^{\frac{1}{\Theta}}$ \\
\hline $\begin{array}{l}\text { Péter and } \\
\text { Fazekas } \\
\text { (2014) (en- } \\
\text { vironmental } \\
\text { param- } \\
\text { eterization } \\
\text { model) }\end{array}$ & $V(\rho, \underline{e})=\frac{e_{4} V_{\text {Max }}}{e_{3}+e_{2}\left(\frac{\rho}{1-\rho^{e_{5}}}\right)^{e_{1}}}$ \\
\hline
\end{tabular}


It was necessary to review the evolution of the velocity-density laws in the literature, since these functions have a decisive role in the fundamental equation in case of traffic models. They shall satisfy two conflicting needs: to be as simple as possible, so that the large complex simulation models provide fast numerical calculations, while satisfying the real practical situations as widely as possible. A common feature of the velocity-density laws is that they result from non-linear regression analysis based on measurements and hypotheses (preconceptions). The common flaw of the classical results is that because they do not refer to other environmental impacts, they only examine the effect of speed on the vehicle density, so essentially any of them can be suitable for a given modelling. Péter and Fazekas (2014) goes much further and examines also the environmental parameters. In case of the IDMs the comprehensive examination of the speed-density functions is an interesting problem, because without preconception an expanded set of functions can be examined from the point of view of which known function types or other new models are considered as valid by this model. Of course, neither the classic IDMs contain the environmental parameters mentioned above, so the studies in this area are far from closed.

The next section is dedicated to generate microscopic and macroscopic simulated traffic data, which are the density and the velocity. In addition, the impact of microscopic parameters to macroscopic ones is investigated.

\section{Macroscopic Traffic Data Generation}

Based on microscopic traffic simulation, density-time and velocity-time functions will be computed in this section.

Subsection 2.1 presents the simulation assumptions and the IDM intended to represent the microscopic longitudinal vehicle motion. Subsection 2.2 introduces the used mathematical formula to compute macroscopic parameters (velocity and density). Subsection 2.3 presents the simulation results and discussions and subsection 2.4 studies the impact of microscopic parameter on macroscopic ones.

\subsection{Microscopic Traffic Model: Intelligent Driver Model}

The IDM is an Adaptive Cruise Control (ACC) system intended for adjusting the driver's longitudinal desired velocity and safety time gap. The IDM, developed by Kesting et al. (2008), is expressed by:

$$
\dot{v}_{n}=a_{n}\left(1-\left(\frac{v_{n}}{v_{n}^{0}}\right)^{4}-\left(\frac{s^{*}\left(v_{n}, \Delta_{v_{n}}\right)}{s_{n}}\right)^{2}\right),
$$

where: $a_{n}$ is the maximum acceleration of the vehicle $n$ $\left[\mathrm{m} / \mathrm{s}^{2}\right] ; v_{n}^{0}$ the desired velocity of the vehicle $n[\mathrm{~m} / \mathrm{s}] ; s_{n}$ the distance gap $[\mathrm{m}]$ :

$$
s_{n}=\Delta x_{n}-l_{n+1} \text {. }
$$

The desired minimum gap of the vehicle $n, s_{n}^{*}$ is given by:

$$
s^{*}\left(v_{n}, \Delta v_{n}\right)=s_{n}^{0}+T_{n} v_{n}-\frac{v_{n} \Delta v_{n}}{2 \sqrt{a_{n} v_{n}}},
$$

where: $a_{n}$ is the desired deceleration of the vehicle $n\left[\mathrm{~m} / \mathrm{s}^{2}\right] ; s_{n}^{0}$ the jam distance of the vehicle $n[\mathrm{~m}] ; T_{n}$ the safety time gap of the vehicle $n$ [s].

Compared to the other Adaptive Cruise Control (ACC) models in the literature, the IDM shows more advantages in terms of easy implementation, calibration and the intuitive and the availability of its parameters. These ones are the desired velocity $v_{n}^{0}$ and the safety time gap $T_{n}$, which are fixed as system inputs

\subsection{Density and Velocity Formula}

\subsubsection{Density Formula}

As shown in subsection 1.15 in Table 2, the quotient between the traffic density and the jam density are used in all models in the literature for density normalization. In this paper, the density is already normalized. Here, the traffic road section is limited by the first and the last vehicle. Then, the density formula can be written as:

$$
d(t)=\frac{\sum_{k=1}^{N} l_{k}}{L_{\text {platoon }}(t)},
$$

where: $l_{k}$ is the length of the vehicle $(k=1,2, \ldots, N) ; N$ the number of vehicle in the platoon; $L_{\text {platoon }}$ the length of the platoon. The density $d$ can be normalized in the interval $[0,1]$. Then, we have:

$$
d=\frac{D}{D_{\max }},
$$

where: $D_{\max }=1$ in this case. For this purpose, in the rest of this paper, the density $d$ will be noted as $D$.

\subsubsection{Velocity Formula}

The platoon velocity $\mathrm{v}$ is the average velocity of the vehicles in the platoon which is given by:

$$
v(t)=\frac{1}{N} \sum_{n=1}^{N} v_{n}(t),
$$

where: $v_{n}$ is the velocity of the vehicle $n$.

\subsection{Simulations and Results}

\subsubsection{The Simulation Model}

The following system of non-linear matrix differential equations shows the structure of the IDM under investigation. For a multi vehicle system, the IDM is given by:

$$
\langle\underline{\underline{A}}\rangle^{-1} \ddot{x}(t)+\langle\underline{\underline{V}}\rangle^{-1} \underline{f_{1}}(\dot{x}(t))+\langle\underline{\underline{S}}\rangle \underline{f_{2}}(x(t))=\underline{1}
$$

with

$$
\langle\underline{\underline{A}}\rangle^{-1}=\left\langle\frac{1}{a_{1}}, \frac{1}{a_{2}}, \ldots, \frac{1}{a_{N}}\right\rangle
$$




$$
\begin{aligned}
& \langle\underline{\underline{V}}\rangle^{-1}=\left\langle\frac{1}{v_{1}^{4}}, \frac{1}{v_{2}^{4}}, \ldots, \frac{1}{v_{N}^{4}}\right\rangle ; \\
& \langle\underline{\underline{S}}\rangle=\left\langle s_{1}, s_{2}, \ldots, s_{N}\right\rangle, \\
& s_{i}=s_{0 i}=\text { fixed or: } \\
& s_{i}=s_{i}\left(\dot{x}_{i-1}, \dot{x}_{i}\right),(i=1,2, \ldots, N) .
\end{aligned}
$$$$
\underline{f_{1}}(\dot{x}(t))=\left[\begin{array}{c}
\dot{x}_{1}^{4} \\
\dot{x}_{2}^{4} \\
\ldots \\
\dot{x}_{N}^{4}
\end{array}\right] \text {; }
$$$$
\underline{f_{2}}(x(t))=\left[\begin{array}{c}
\frac{1}{\left(x_{0}-x_{1}\right)^{2}} \\
\frac{1}{\left(x_{1}-x_{2}\right)^{2}} \\
\cdots \\
\frac{1}{\left(x_{n-1}-x_{N}\right)^{2}}
\end{array}\right] ;
$$$$
\underline{1}=\left[\begin{array}{c}
1 \\
1 \\
\cdots \\
1
\end{array}\right] .
$$

This model examines the longitudinal dynamics in the direction of travelling in case of vehicles travelling in a lane and is specifically capable of analysing the evolution of the speed-density law. The movement is performed on a line, which is a given lane. We have dealt with the further generalization of the model in Derbel et al. (2012b, 2013).

\subsubsection{Simulation Assumptions}

In our simulations, the following assumptions are taken into account:

- the AAC model, which is our microscopic traffic model, is already validated and its parameters are identified based on experimental data (Kesting et al. 2008);

- the traffic is homogeneous. It means that the longitudinal motion of all vehicles is controlled by the same ACC model (IDM) with the same parameter values; a heterogeneous traffic includes vehicles with manual and automatic driving modes;

- the road has only one lane.

- only the longitudinal vehicle motion is studied; the lane change and the lateral motion are neglected.

\subsubsection{The Simulator}

The development of traffic simulators has received much attention in recent years. Many companies have invested in such projects and have made simulators integrating various dynamic and kinematic models of vehicles, driver and road architectures available. These simulators are known by their complexities in terms of software development. For example, the simulator ARCHISIM adopts multi-agent approach and techniques from artificial intelligence to simulate complex phenomena.

Our motivations for the development of a mixed traffic simulator (coexistence of automated and manual driving style in the same traffic section) are justified according to the following two aspects:

- the high cost of traffic simulators in the market;

- the collision management problem. For example, the traffic simulators CarMaker and Microsimulation of Road Traffic Flow (MRTF) do not perfectly manage collisions between vehicles.

Our mixed traffic simulator is developed using the $\mathrm{C}$ language and the Open Graphics Library (OpenGL) library for graphical interface. Fig. 3 shows the structure of mixed traffic simulator. This simulator has inputs that are used by its different modules to save the output of the simulation in different formats such as video and/or data required for post-processing.

The inputs of the simulator are the following three:

- 'XML File' in which some parameters can be set: the simulation number, the initial vehicle number, the road length and the minimum and maximum percentage of automated vehicles;

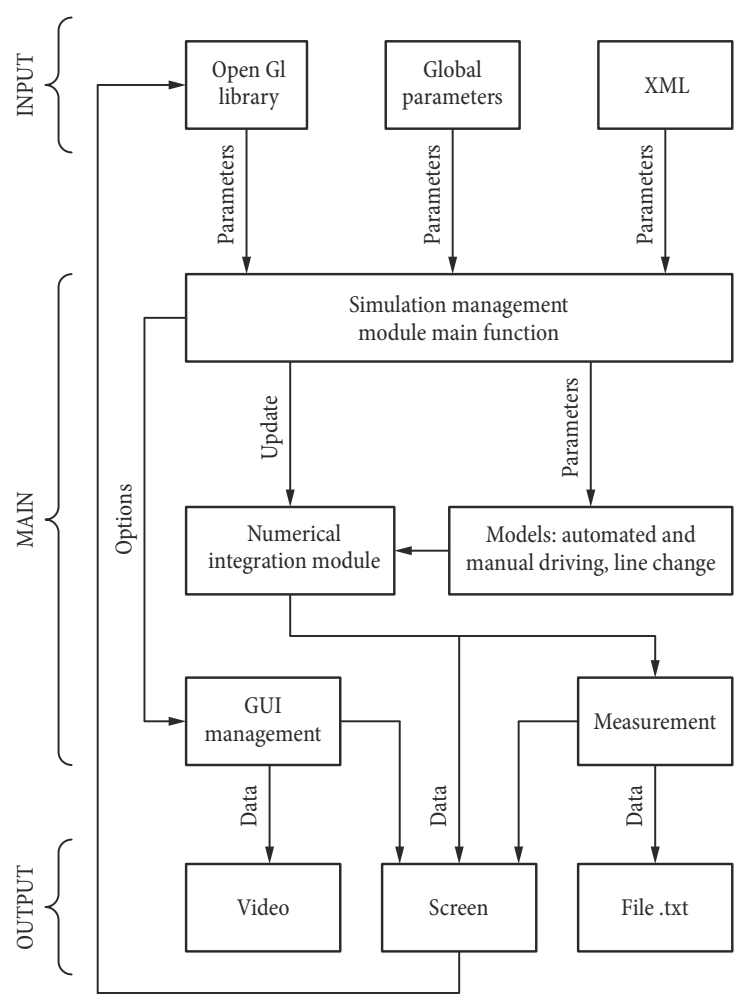

Fig. 3. Structure of the developed simulator 
- 'Global Settings': these parameters are fixed throughout the simulation (e.g. road width);

- 'OpenGL library': this library is responsible for the Graphical User Interface (GUI) management such as scenarios and architectures used in the simulation.

In the simulator body, the following modules are developed:

- 'Scenario Development Module': This module includes the different developed scenarios. They take their input parameters through the 'main' function. It selects the script to run from the choice made in the XML file. In this module, there are useful models: the IDM for longitudinal automated driving style, the Two Velocity Difference Model (TVDM) (Ge et al. 2008) for longitudinal manual driving style and the Minimizing Overall Braking change Induced by Lane change (MOBIL) model (Kesting et al. 2008) for the lane change management. These models need to be updated in each simulation step through the numerical integration module (Runge Kutta);

- 'GUI Management Module': This module is based on the OpenGL library and the measurements are recorded to generate and update the simulator graphical interface.

The outputs of this simulator are the following three:

- 'Screen' - displays the architecture and the scenario;

- 'Video' - to save the simulation;

- 'File .txt or .bin' - saves the data needed for postprocessing.

Fig. 4 shows the position of the vehicles in a platoon.

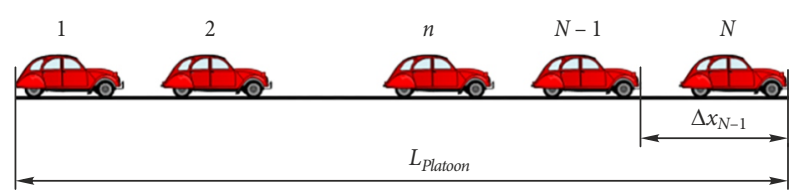

Fig. 4. Positions of the vehicles in the platoon

The IDM simulation model with the parameters given by Table 3 is applied to simulate the fully automated traffic using our developed microscopic traffic simulator. The presented parameter values in this table are originated from an identification step using experimental data and used to validate the IDM with the fundamental diagram by Kesting et al. (2008). At each simulation step, the simulated microscopic traffic data such as the position, the velocity and the acceleration of each vehicle are computed. Then, the density and the velocity are computed according to Eqs (17) and (18) respectively.

Simulation parameters are given in Table 4. In this table, the jam distance $s_{n}^{0}$ is set to zero in order to have the maximum density equal to 1 .
Table 3. IDM simulation parameters

\begin{tabular}{|l|c|c|}
\hline \multicolumn{1}{|c|}{ Parameter } & Mean value & Unit \\
\hline Maximum acceleration $a_{n}$ & 3 & $\mathrm{~m} / \mathrm{s}^{2}$ \\
\hline Desired deceleration $b_{n}$ & 3 & $\mathrm{~m} / \mathrm{s}^{2}$ \\
\hline Safety time headway $T_{n}$ & 1.5 & $\mathrm{~s}$ \\
\hline
\end{tabular}

Table 4. Simulation parameters

\begin{tabular}{|l|c|c|}
\hline \multicolumn{1}{|c|}{ Parameter } & Value & Unit \\
\hline Vehicle number & 20 & vehicle \\
\hline Simulation time & 1000 & $\mathrm{~s}$ \\
\hline Simulation time step & 0.01 & $\mathrm{~s}$ \\
\hline Initial inter distance & 0 & $\mathrm{~m}$ \\
\hline Initial acceleration & 0 & $\mathrm{~m} / \mathrm{s}^{2}$ \\
\hline Desired velocity & 50 & $\mathrm{~km} / \mathrm{h}$ \\
\hline Initial velocity & 0 & $\mathrm{~km} / \mathrm{h}$ \\
\hline The jam distance & 0 & $\mathrm{~m}$ \\
\hline Vehicle length & 5 & $\mathrm{~m}$ \\
\hline
\end{tabular}

To study the sensitivity of macroscopic parameters (velocity and density) according to the microscopic parameters $\left(a_{n}, b_{n}, T_{n}\right)$, traffic simulation is performed made for each of the maximum acceleration $a_{n}$ values (the other microscopic parameters are constants), the desired deceleration $b_{n}$ (the other microscopic parameters are constants) and the safety time headway $T_{n}$ (the other microscopic parameters are constants).

\subsubsection{Simulation Results}

Fig. 5 shows the density versus the time (first curve) and the velocity versus the time (second curve) during $1000 \mathrm{~s}$ of simulation with the microscopic parameters given by Table 3 and the simulation parameters given by Table 4 . Here as the density increases, the velocity decreases until the stable state.
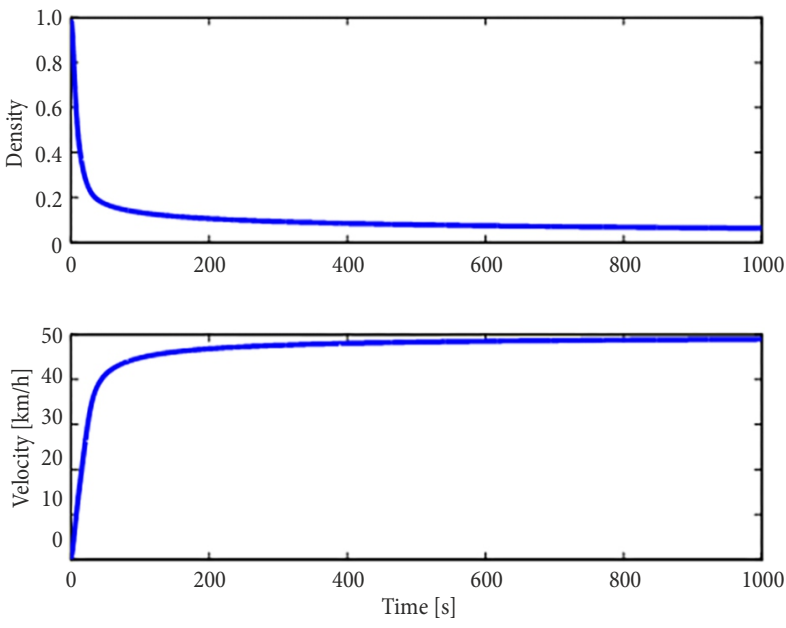

Fig. 5. Traffic density and velocity time functions 


\subsection{From Macroscopic Parameters to Microscopic Parameters}

In this section, two goals are fixed: the first one consists in studying the dependency of the shape of the velocity-time and the density-time functions to microscopic parameters. The second goal is to study the ability to identify microscopic parameters from macroscopic parameters. Microscopic parameters are the maximum acceleration $a_{n}$, the desired deceleration $b_{n}$ and the safety time headway $T_{n}$. In this step the following assumptions are made: the maximum acceleration $\mathrm{a}_{n} \in[2,7] \mathrm{m} / \mathrm{s}^{2}$ the desired deceleration $\mathrm{b}_{n} \in[2,5] \mathrm{m} / \mathrm{s}^{2}$ and the safety time headway $\mathrm{T}_{n} \in[1.5,4] \mathrm{s}$. The ranges are empirical intervals given by Kesting et al. (2008). In this subsection, it is noted that all vehicles have the same parameters (homogeneous traffic).

\subsubsection{Impact of $a_{n}, b_{n}$ and $T_{n}$ on Density}

As shown in Fig. 6, the maximum acceleration and the desired deceleration have no impact on traffic density.
Indeed, when the traffic becomes stable:

$$
\Delta v_{n}=0, \forall n \in\{1, \ldots, N\}
$$

and then

$$
\frac{v_{n} \Delta v_{n}}{2 \sqrt{a_{n} v b_{n}}}=0
$$

The safety time headway $T_{n}$ has an impact on traffic density i.e when $T_{n}$ increases, traffic density decreases. In fact, vehicles tend to increase inter-distance when $T_{n}$ is high, then the traffic density decreases.

\subsubsection{Impact of $a_{n}, b_{n}$ and $T_{n}$ on Velocity}

As shown in Fig. 7, the maximum acceleration and the desired deceleration have no impact on the velocity. The same reason cited in 3.4 is the cause of this result: when the traffic becomes stable, $\Delta v_{n}=0 \forall n \in\{1, \ldots, N\}$ and then $\frac{v_{n} \Delta v_{n}}{2 \sqrt{a_{n} v b_{n}}}=0$. The safety time headway $T_{n}$ has an impact on the velocity i.e. when $T_{n}$ decreases vehicles ve-
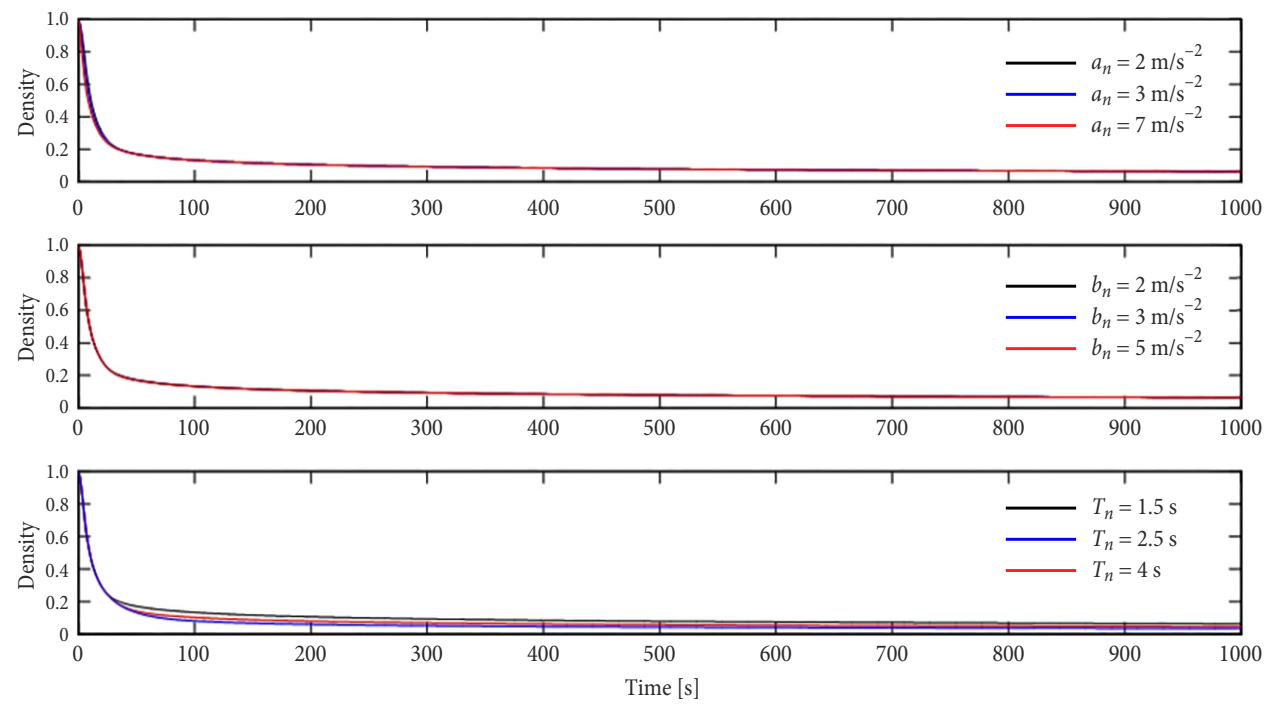

Fig. 6. Density for different $a_{n}, b_{n}$ and $T_{n}$ sets
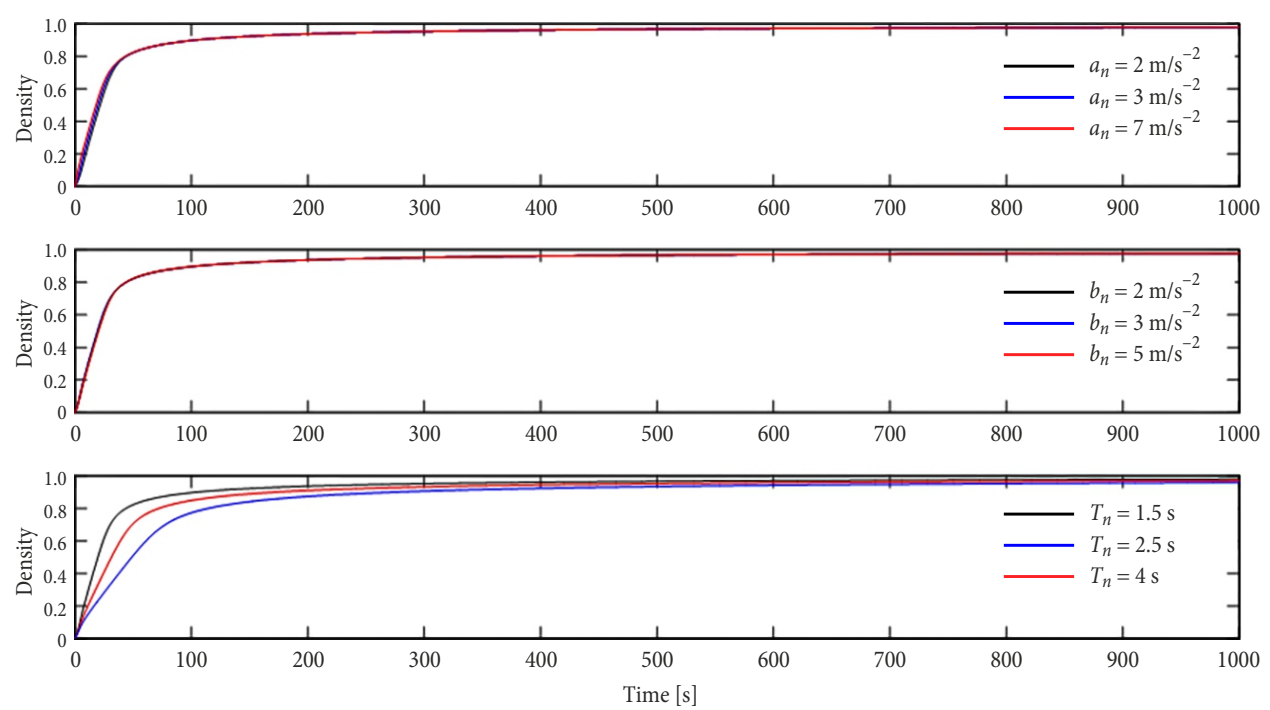

Fig. 7. Velocity for different $a_{n}, b_{n}$ and $T_{n}$ sets 


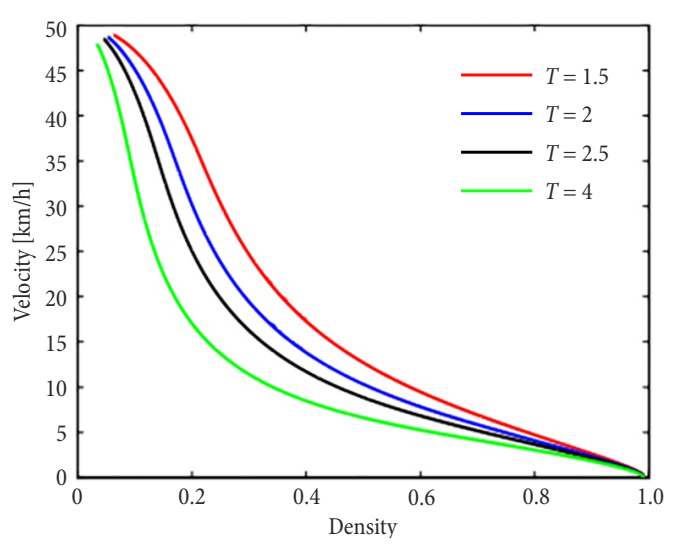

Fig. 8. Impact of the time headway on the velocity-density curve

locity increases. Vehicles accelerate to reach the desired velocity until the safety time headway is guaranteed.

Fig. 8 shows the impact of the time headway $T_{n}$ on the macroscopic parameters. Now the curves of this figure will be considered as reference to us (velocitydensity map). In case of unknown time headway, from the density and the velocity values, we can identify the mean value of time headway between vehicles by mean of interpolation.

\section{New Velocity-Density Model Synthesis Method}

The new method consists in studying the variation of each of the two macroscopic parameters (velocity and density given by the Figs 6 and 7 separately along the simulation time. To carry out this study, a family of functions is computed to approximate the density-time and velocity-time functions. For a given $\gamma$, this family of candidate functions is expressed by:

$H_{\gamma}=\left\{F / \exists\left(F_{1}, F_{2}, \tau_{f}\right) \in \mathfrak{R}^{3} ; F(t)=F_{1} \exp \left(-\frac{t^{\gamma}}{\tau_{f}}\right)+F_{2}\right\}$.

For reasons, which will be later explained, $\gamma$ is fixed for all candidate functions of the two macroscopic parameters.
The function $D \in H_{\gamma}$, which is a candidate to approximate the density-time function, is given by:

$$
D(t)=D_{1} \exp \left(-\frac{t \gamma}{\tau_{d}}\right)+D_{2}
$$

where: $D_{1}, \tau_{\mathrm{d}}$ and $D_{2}$ are constants.

The function $v \in H_{\gamma}$, which is a candidate to approximate the velocity-time function, is given by:

$$
v(t)=V_{1} \exp \left(-\frac{t^{\gamma}}{\tau_{v}}\right)+V_{2},
$$

where: $V_{1}<0$, and $\tau_{v}>0, V_{2}>0$ are constants.

$\gamma$ is fixed for the two candidate functions to have an analytical time independent velocity-density relationship from Eqs (20) and (21). D and $v$ functions are used to fit separately the density and the velocity data in the least square sense. The fitted curves of the density and the velocity together with the macroscopic data given by IDM simulation. Parameters of these two functions, obtained with setting $\gamma=0.8$, are summarized in Table 5 . The mean, the maximum and the minimum absolute errors are given by Table 6 . The absolute error between data given by microscopic simulation and the computed functions $D$ and $v$ is small compared to the maximum values of each one. The IDM is written as:

and

$$
\dot{v}_{n}=a_{n}\left(1-\left(\frac{v_{n}}{v_{n}^{0}}\right)^{4}-\left(\frac{s^{*}\left(v_{n}, \Delta v_{n}\right)}{s_{n}}\right)^{2}\right)
$$

$$
s^{*}\left(v_{n}, \Delta v_{n}\right)=s_{n}^{0}+T_{n} v_{n}-\frac{v_{n} \Delta v_{n}}{2 \sqrt{a_{n} v_{n}}} .
$$

The solution for the velocity is given by:

$$
v(t)=V_{1} \exp \left(-\frac{t^{\gamma}}{\tau_{v}}\right)+V_{2} \text {. }
$$

$$
\frac{-t^{\gamma}}{\tau_{v}} \rightarrow 0 \text {. }
$$
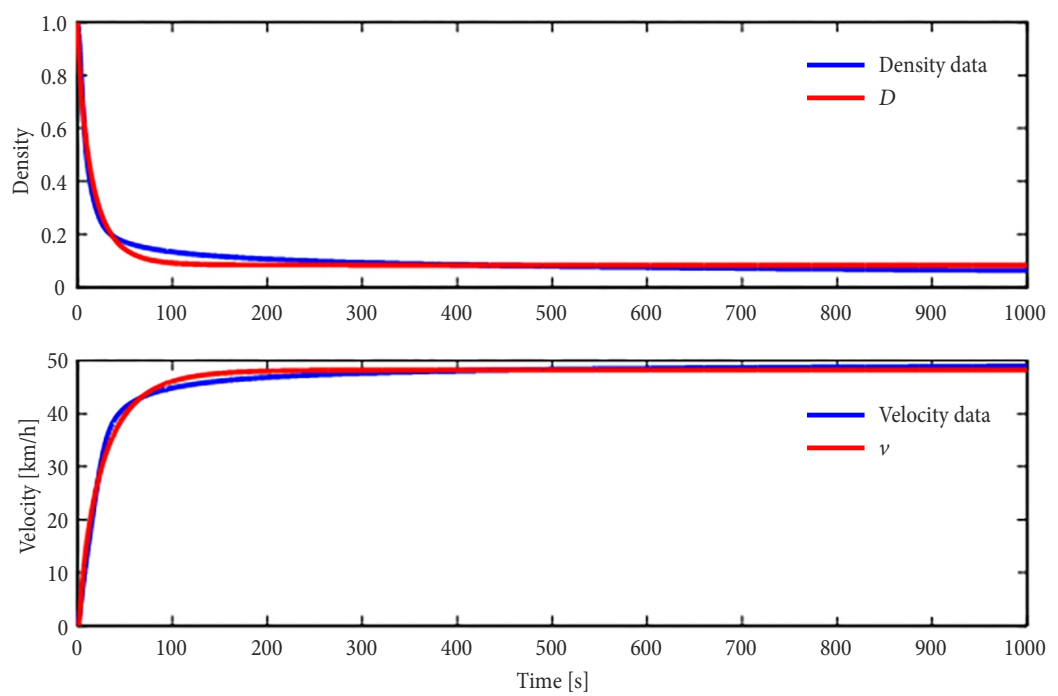

Fig. 9. Density-time function with $D$ function and velocity-time function with $v$ function 
Then,

$$
\left(\frac{T_{n}}{s_{n}}\right)^{2}\left(v_{n}^{0}\right)^{4} V_{2}^{2}+V_{2}^{4}=\left(v_{n}^{0}\right)^{4}
$$

and, as shown in Eq (22), $V_{2}$ depends on the interdistance and the safety time headway $T_{n}$ and does not depends on $a_{n}$ and $b_{n}$.

Table 5. Parameters of the $D$ and $v$ functions with $\gamma=0.8$

\begin{tabular}{|l|c|c|}
\hline \multicolumn{1}{|c|}{ Parameter } & Mean value & Unit \\
\hline$D_{1}$ & 0.8971 & - \\
\hline$\tau_{d}$ & 0.1205 & $\mathrm{~s}$ \\
\hline$D_{2}$ & 0.1029 & - \\
\hline$V_{1}$ & -48.4583 & $\mathrm{~km} / \mathrm{h}$ \\
\hline$\tau_{v}$ & 0.0813 & $\mathrm{~s}$ \\
\hline$V_{2}$ & 48.48205 & $\mathrm{~km} / \mathrm{h}$ \\
\hline$R^{2}$ (velocity) & 0.9788 & - \\
\hline$R^{2}$ (density) & 0.9399 & - \\
\hline
\end{tabular}

Table 6. Density and velocity error

\begin{tabular}{|l|c|c|c|}
\hline \multicolumn{1}{|c|}{ Parameter } & Mean & Max & Min \\
\hline Density error & 0.0157 & 0.0786 & 0 \\
\hline Mean velocity error & 0.6402 & 6.3786 & 0 \\
\hline
\end{tabular}

\section{Velocity-Density Model Synthesis}

From the density model defined by Eq. (20) and the velocity model defined by Eq. (21), the VDM is developed analytically in this section.

\subsection{Model Synthesis}

Eq. (20) is given by:

$$
D(t)=D_{1} \exp \left(-\frac{t^{\gamma}}{\tau_{d}}\right)+D_{2} .
$$

The function $v \in H_{\gamma}$, which is a candidate to approximate the velocity-time function, is given by:

$$
v(t)=V_{1} \exp \left(-\frac{t^{\gamma}}{\tau_{v}}\right)+V_{2} .
$$

From these two equations, we get:

$$
\begin{aligned}
& t^{\gamma}=\tau_{d} \ln \left(\frac{D(t)-D_{2}}{D_{1}}\right) ; \\
& t^{\gamma}=\tau_{v} \ln \left(\frac{v(t)-V_{2}}{V_{1}}\right) ; \\
& v(D(t))=V_{1}\left(\frac{D(t)-D_{2}}{D_{1}}\right)^{\frac{\tau_{d}}{\tau_{v}}}+V_{2} .
\end{aligned}
$$

Let $V$, the function defined as:

$$
V: D \rightarrow V_{1}\left(\frac{D(t)-D_{2}}{D_{1}}\right)^{\frac{\tau_{d}}{\tau_{v}}}+V_{2}
$$

with slight abuse of notation, we have $v(D(t))=V(D)$.

Then, the analytic velocity-density relationship can be written as:

$$
v(D)=V_{1}\left(\frac{D-D_{2}}{D_{1}}\right)^{\frac{\tau_{d}}{\tau_{v}}}+V_{2} .
$$

To calibrate this model, the limit conditions are applied in the next subsection.

\subsection{Generalized Velocity-Density Model}

Applying the limit conditions given by:

$$
\left\{\begin{array}{l}
D=1 \Rightarrow V=\varepsilon_{V} \approx 0 ;\left(\varepsilon_{V}=V_{2}+V_{1}\right) ; \\
D=D_{2} \Rightarrow V=V_{\max }
\end{array}\right.
$$

to Eq. (28), we have:

$$
\left\{\begin{array}{l}
V_{1}\left(\frac{1-D_{2}}{D_{1}}\right)^{\frac{\tau_{d}}{\tau_{v}}}+V_{2}=\varepsilon_{V} \\
V_{1}\left(\frac{D_{2}-D_{2}}{D_{1}}\right)^{\frac{\tau_{d}}{\tau_{v}}}+V_{2}=V_{\max }
\end{array}\right.
$$

Eq. (30) is given by Eqs (31) and (32):

$$
\begin{aligned}
& V_{2}=V_{\max } ; \\
& V_{1}=\frac{-V_{\max }}{\left(\frac{1-D_{2}}{D_{1}}\right)^{\frac{\tau_{d}}{\tau_{v}}}}+\frac{\varepsilon_{V}}{\left(\frac{1-D_{2}}{D_{1}}\right)^{\frac{\tau_{d}}{\tau_{v}}}}
\end{aligned}
$$

using Eqs (28), (31) and (32):

$$
V(D)=\left(\frac{-V_{\max }}{\left(\frac{1-D_{2}}{D_{1}}\right)^{\frac{\tau_{d}}{\tau_{v}}}}+\frac{\varepsilon_{V}}{\left(\frac{1-D_{2}}{D_{1}}\right)^{\frac{\tau_{d}}{\tau_{v}}}}\right)\left(\frac{D-D_{2}}{D_{1}}\right)^{\frac{\tau_{d}}{\tau_{v}}}+V_{\max } .
$$

Then, the generalized VDM can finally be expressed as:

$$
V(D)=V_{\max }\left(1-\left(\frac{D-D_{2}}{1-D_{2}}\right)^{\frac{\tau_{d}}{\tau_{v}}}\right)+\varepsilon_{V}\left(\frac{D-D_{2}}{1-D_{2}}\right)^{\frac{\tau_{d}}{\tau_{v}}},
$$

where: $\tau_{d}>0, \tau_{v}>0, D \in\left[1, D_{2}\right], D_{1}>0, D_{2}>0$ are constants, and $D_{1}+D_{2}=1$. From this calibrated model, some of models defined in section 1 can be derived. Next subsections show which of these models can be obtained.

\subsection{Sub Models}

From the model defined by Eq. (34), some of existing models in the literature can be derived by fixing some of its parameters:

$$
\text { - If } D_{2}=0 \text { and } \varepsilon_{V}=0 \text {. }
$$


In this case,

$$
V(D)=V_{\max }(1-D)^{\frac{\tau_{d}}{\tau_{v}}}
$$

represents the Pipes model given by Eq. (6) where $r=1$, $\varepsilon_{V}=0, m=\frac{\tau_{d}}{\tau_{v}}$ and $D_{\max }=1$. One of the advantages of the new model is the finding of the physical meaning of the parameter $m$.

- If $D_{2}=0, \varepsilon_{V}=0$ and $\tau_{d}=\tau_{v}$.

In this case,

$$
V(D)=V_{\max }(1-D) \text {. }
$$

Eqs (20) and (21) can be written as:

$$
\begin{aligned}
& D(t)=D_{1} \exp \left(-\frac{t^{\gamma}}{\tau_{d}}\right)+D_{2} ; \\
& V(t)=V_{1} \exp \left(-\frac{t^{\gamma}}{\tau_{v}}\right)+V_{2} .
\end{aligned}
$$

Then:

$$
V(D)=V_{1}\left(\frac{D}{D_{1}}-1\right)+V_{2} \text {, }
$$

where: the relationship between velocity and density remains linear. In case of the Greenshields model, $V_{\text {max }}=V_{2}$ and $D_{\max }=1$.

- If $D_{2}=0, \varepsilon_{V}=0$ and $\frac{\tau_{d}}{\tau_{v}}=\frac{p+1}{2}$ and $p \geq-1$.

In this case,

$$
V(D)=V_{\max }\left(1-D^{\frac{p+1}{2}}\right)
$$

which is the Drew model given by Eq. (7), where $D_{\max }=1$. Since $\tau_{d}$ and $\tau_{v}$ are two time constants, then $\frac{\tau_{d}}{\tau_{v}} \geq 0$.

Therefore, the choice of $\frac{\tau_{d}}{\tau_{v}}=\frac{p+1}{2}$ leads to have $p+1 \geq 0$ and then $p \geq-1$. In this study, the density is expressed as:

$$
D(t)=D_{1} \exp \left(-\frac{t^{\gamma}}{\tau_{d}}\right)+D_{2} .
$$

It implies that when $t \rightarrow \infty, D_{1} \exp \left(-\frac{t^{\gamma}}{\tau_{d}}\right)<<D_{2}$.

If $D_{2} \rightarrow 0$, then only lower densities can be predicted. Therefore, velocity can be estimated only in free flow condition. Thus, the model cannot predict lower velocities. This result has been proven by Ardekani et al. (2011), empirically and proved analytically now by our method of velocity-density synthesis and through our generic model.

\section{Conclusions}

The velocity-density functions known from the literature are results of non-linear regressions, which are obtained by the determination of functions that fit the measured coherent speed-density values with the minimum error.
The first step of the analysis we have performed in the field of IDMs is also a non-linear regression, which was developed based on the speed and density changes over time. Next, we have described a direct function relation for the speed-density function using a time parameter.

There is no defined speed-density law in the IDM, so it was an important achievement to show what speeddensity function is followed by the IDM.

The method presented in this publication has been developed for the determination of the speed-density function $V(D)$ specific to the IDM, which took into account the development of the process of the free flow.

The developed method is based on the regression procedure relative to the parametric functions $V(t)$ and $D(t)$. The selection of the proper function type was performed using regression analysis (minimization of expected error, $\chi^{2}$ analysis) with the boundary condition that the defined function $V(D)$ should be mathematically easily tractable. The $\left[\left(V_{i}, t_{i}\right) ;\left(D_{i}, t_{i}\right), i=1,2, \ldots, n\right]$ set of pairs of points constituting the basis of the regression was provided by the IDM simulation.

In the case of the function $V(D)$ the studies presented aimed, therefore, at the definition of a general mathematical form and thus $V_{\max }$ was a general parameter as well. (In the publication, in a particular example the value of that was $50 \mathrm{~km} / \mathrm{h}$, which is typical of urban traffic.) A separate new interesting result is that the law presented by us has defined a more general class of function compared to what was known earlier. At the same time this has also integrated three known speed-density relationships as special cases (with the proper selection of parameters) (Greenshields 1935; Pipes 1967; Drew 1968).

We emphasize that the analysis - in terms of the development of simulation models - led to another important question. The known speed-density functions are univariate functions of the vehicle densities. The significant formal differences between these types of functions raise the question whether the differences can significantly be predetermined by the environmental parameters, as well. An interesting question is whether more general classes of functions that integrate more known function types can be determined by broader measurement and analysis, and further IDM analysis. These known function types are multivariate functions that depend on the density and environmental parameters, as well, for example the one presented in Péter and Fazekas (2014). The simulator discussed in this article is capable of carrying out these further investigations.

It is an exciting issue, that the parameters $D_{1}, D_{2}$, $V_{1}, V_{2}, \tau_{d}, \tau_{v}$ acting in the function $V(D)$ are other functions, as they depend on the parameters of the IDM. The research is, therefore, not closed, the topic of further really interesting studies is how these parameters depend on the parameters of the IDM and on their distribution.

Similarly, another important area is the investigation of environmental parameters that affect the IDM parameters. 
Taking into account the velocity processes the method is directly connectable with the optimization of environmental pollutions, so further research in this direction may be conducted within the project. It has an important influence in many areas of transportation (Tettamanti et al. 2015; Gumz, Török 2015; Török et al. 2015; Lakatos 2015; Szauter et al. 2014).

\section{Acknowledgements}

Authors thank the Hungarian Government and the European Social Fund for their financial support to the 'Smarter Transport' - IT for co-operative transport system project TÁMOP-4.2.2.C-11/1/KONV-2012- 0012.

\section{References}

Ardekani, A. S.; Ghandehari, M.; Nepal, M. S. 2011. Macroscopic speed-flow models for characterization of freeway and managed lanes, Bulletin of the Polytechnic Institute of Iasi 7: 149-160.

Bando, M.; Hasebe, K.; Nakanishi, K.; Nakayama, N. 1998. Analysis of optimal velocity model with explicit delay, Physical Review E: Covering Statistical, Nonlinear, Biological, and Soft Matter Physics 58(5): 5429-5435. https://doi.org/10.1103/PhysRevE.58.5429

Bede, Z.; Péter, T. 2014. Optimal control with the dynamic change of the structure of the road network, Transport 29(1): 36-42. https://doi.org/10.3846/16484142.2014.895959

Bede, Z.; Péter, T.; Szauter, F. 2013. Variable network model, IFAC Proceedings Volumes 46(25): 173-177. https://doi.org/10.3182/20130916-2-TR-4042.00026

Del Castillo, J. M. 2012. Three new models for the flow-density relationship: derivation and testing for freeway and urban data, Transportmetrica 8(6): 443-465. https://doi.org/10.1080/18128602.2011.556680

Del Castillo, J. M.; Benítez, F. G. 1995. On the functional form of the speed-density relationship - I: general theory, Transportation Research Part B: Methodological 29(5): 373-389. https://doi.org/10.1016/0191-2615(95)00008-2

Derbel, O.; Mourllion, B.; Basset, M. 2012a. Extended safety descriptor measurements for relative safety assessment in mixed road traffic, in 2012 15th International IEEE Conference on Intelligent Transportation Systems (ITSC), 16-19 September 2012, Anchorage, Alaska, US, 752-757. https://doi.org/10.1109/ITSC.2012.6338774

Derbel, O.; Péter, T.; Zebiri, H.; Mourllion, B.; Basset, M. 2012b. Modified intelligent driver model, Periodica Polytechnica Transportation Engineering 40(2): 53-60. https://doi.org/10.3311/pp.tr.2012-2.02

Dömötörfi, Á.; Péter, T.; Szabó, K. 2016. Mathematical modeling of automotive supply chain networks, Periodica Polytechnica Transportation Engineering 44(3): 181-186. https://doi.org/10.3311/PPtr.9544

Derbel, O.; Peter, T.; Zebiri, H.; Mourllion, B.; Basset, M. 2013. Modified intelligent driver model for driver safety and traffic stability improvement, IFAC Proceedings Volumes 46(21): 744-749.

https://doi.org/10.3182/20130904-4-JP-2042.00132

Drake, J. S.; Schofer, J. L.; May, A. D. 1967. A statistical analysis of speed-density hypotheses in vehicular traffic science, Highway Research Record 154: 112-117.

Drew, D. R. 1968. Traffic Flow Theory and Control. McGrawHill Inc. 467 p.
Ge, H. X.; Cheng, R. J.; Li, Z. P. 2008. Two velocity difference model for a car following theory, Physica A: Statistical Mechanics and its Applications 387(21): 5239-5245. https://doi.org/10.1016/j.physa.2008.02.081

Greenberg, H. 1959. An analysis of traffic flow, Operations Research 7(1): 79-85. https://doi.org/10.1287/opre.7.1.79

Greenshields, B. D. 1935. A study of traffic capacity, Highway Research Board Proceedings 14: 448-477.

Gumz, F.; Török, Á. 2015. Investigation of cordon pricing in Budakeszi, Periodica Polytechnica Transportation Engineering 43(2): 92-97. https://doi.org/10.3311/PPtr.7579

Helbing, D.; Tilch, B. 1998. Generalized force model of traffic dynamics, Physical Review E: Covering Statistical, Nonlinear, Biological, and Soft Matter Physics 58(1): 133-138. https://doi.org/10.1103/PhysRevE.58.133

Herty, M.; Klar, A. 2003. Modeling, simulation, and optimization of traffic flow networks, SIAM Journal on Scientific Computing 25(3): 1066-1087. https://doi.org/10.1137/S106482750241459X

Holden, H.; Risebro, N. H. 1995. A mathematical model of traffic flow on a network of unidirectional roads, SIAM Journal on Mathematical Analysis 26(4): 999-1017. https://doi.org/10.1137/S0036141093243289

Kesting, A. 2008. Microscopic Modeling of Human and Automated Driving: Towards Traffic-Adaptive Cruise Control: Doctoral Thesis. Faculty of Traffic Sciences, Dresden University of Technology, Germany. 218 p.

Kesting, A.; Treiber, M.; Schönhof, M.; Helbing, D. 2008. Adaptive cruise control design for active congestion avoidance, Transportation Research Part C: Emerging Technologies 16(6): 668-683. https://doi.org/10.1016/j.trc.2007.12.004

Lakatos, I. 2015. Development of a new method for comparing the cold start- and the idling operation of internal combustion engines, Periodica Polytechnica Transportation Engineering 43(4) 225-231. https://doi.org/10.3311/PPtr.8087

MacNicholas, M. J. 2008. A simple and pragmatic representation of traffic flow, in Symposium on the Fundamental Diagram: 75 Years (Greenshields 75 Symposium), 8-10 July 2008, Woods Hole, MA, US, 1-17.

Mahnke, R.; Kaupužs, J. 1999. Stochastic theory of freeway traffic, Physical Review E: Covering Statistical, Nonlinear, Biological, and Soft Matter Physics 59(1): 117-125. https://doi.org/10.1103/PhysRevE.59.117

Molina, J. 2005. Commande de l'inter-distance entre deux véhicules: Thèse pour obtenir le grade de docteur. Institut National Polytechnique de Grenoble, France. 164 p. (in French).

Newell, G. F. 1961. Nonlinear effects in the dynamics of car following, Operations Research 9(2): 209-229. https://doi.org/10.1287/opre.9.2.209

Paveri-Fontana, S. L. 1975. On Boltzmann-like treatments for traffic flow: a critical review of the basic model and an alternative proposal for dilute traffic analysis, Transportation Research 9(4): 225-235. https://doi.org/10.1016/0041-1647(75)90063-5

Péter, T. 2012. Modeling nonlinear road traffic networks for junction control, International Journal of Applied Mathematics and Computer Science 22(3): 723-732. https://doi.org/10.2478/v10006-012-0054-1

Peter, T.; Bokor, J.; Strobl, A. 2013. Model for the analysis of traffic networks and traffic modelling of Győr, IFAC Proceedings Volumes 46(25): 167-172. https://doi.org/10.3182/20130916-2-TR-4042.00023 
Péter, T.; Fazekas, S. 2014. Determination of vehicle density of inputs and outputs and model validation for the analysis of network traffic processes, Periodica Polytechnica Transportation Engineering 42(1): 53-61.

https://doi.org/10.3311/PPtr.7282

Péter, T., Lakatos, I.; Szauter, F. 2015. Analysis of the complex environmental impact on urban trajectories, in ASME 2015 International Design Engineering Technical Conferences and Computers and Information in Engineering Conference, 2-5 August 2015, Boston, Massachusetts, US, 9: 1-7 https://doi.org/10.1115/DETC2015-47077

Pipes, L. A. 1967. Car following models and the fundamental diagram of road traffic, Transportation Research 1(1): 21-29. https://doi.org/10.1016/0041-1647(67)90092-5

Prigogine, I.; Herman, R. C. 1971. Kinetic Theory of Vehicular Traffic. Elsevier. 100 p.

Rakha, H.; Gao, Y. 2010. Calibration of Steady-State Car-Following Models Using Macroscopic Loop Detector Data. Final Report VT-2008-01. Virginia Tech Transportation Institute, US. 24 p.

Tettamanti, T.; Milacski, Z. Á.; Lőrincz, A.; Varga, I. 2015. Iterative calibration method for microscopic road traffic simulators, Periodica Polytechnica Transportation Engineering 43(2): 87-91. https://doi.org/10.3311/PPtr.7685

Török, Á; Kiss, Á; Szendrö, G. 2015. Introduction to the road safety situation in Hungary, Periodica Polytechnica Transportation Engineering 43(1): 15-21. https://doi.org/10.3311/PPtr.7510

Treiber, M.; Hennecke, A.; Helbing, D. 2000a. Congested traffic states in empirical observations and microscopic simulations, Physical Review E 62(2): 1805-1824. https://doi.org/10.1103/PhysRevE.62.1805

Treiber, M.; Hennecke, A.; Helbing, D. 2000b. Microscopic simulation of congested traffic, in D. Helbing, H. J. Herrmann, M. Schreckenberg, D. E. Wolf (Eds.). Traffic and Granular Flow'99: Social, Traffic, and Granular Dynamics, 365-376. https://doi.org/10.1007/978-3-642-59751-0_36

Underwood, R. T. 1961. Speed, volume and density relationship, in B. D. Greenshields, H. P. George, N. S. Guerin, M. R. Palmer, R. T. Underwood (Eds.). Quality and Theory of Traffic Flow: a Symposium. Bureau Highway Traffic, Yale University, 141-188.

Van Aerde, M. W. 1995. Single regime speed-flow-density relationship for congested and uncongested highways, in 74th Annual Meeting of the Transportation Research Board, 22-28 January 1995, Washington, DC, US, 1-26.

Wang, H.; Ni, D.; Chen, Q.-Y.; Li, J. 2013. Stochastic modeling of the equilibrium speed-density relationship, Journal of Advanced Transportation 47(1): 126-150.

https://doi.org/10.1002/atr.172 